USITP-95-03

March 1995

\title{
Hamiltonian BRST Quantization of the Conformal Spinning String
}

\author{
by \\ P. Saltsidis 1 \\ ITP \\ University of Stockholm \\ Box 6730, Vanadisvägen 9 \\ S-113 85 Stockholm \\ SWEDEN
}

\begin{abstract}
The BRST quantization of the null spinning string for different number of supersymmetries is given. A null spinning string with manifest space-time conformal invariance is constructed. Its BRST quantization gives negative critical dimensions for $N \neq 0$ and reproduces previous results for $N=0$.
\end{abstract}

\footnotetext{
${ }^{1}$ e-mail address:panos@vanosf.physto.se
} 


\section{Introduction}

The characteristic scale of string theory is given by the string tension $T$. At energies of order of $\sqrt{T}$ or higher, string physics truly distinguishes itself from point particle physics. One may alternatively view the high energy limit as the zero tension limit, since only the energy measured in string units, $\frac{E}{\sqrt{T}}$ is relevant. Strings in which the zero tension $(T \rightarrow 0)$, has been taken are called null and they were first considered by Schild [1]. It has been shown in [2] that a null string corresponds to a collection of particles each of which moves independently along a null geodesic. In this respect the zero tension limit of a string theory is the analogue of massless particles.

The quantization of the null string was firstly discussed in [3] and then in [4]. In [3] was found that the quantization of the string does not give rise to critical dimensions, while in [4 was pointed out that the issue depends crucially on the choice of ordering, and that normal ordering gives a critical dimension $d=26$ for the bosonic null string. In [5], it was shown that when $T \rightarrow 0$, the Weyl invariance is substituted by conformal invariance at the classical level. In [6] using general arguments it is shown that requiring this symmetry to hold at the quantum level leads to restrictions on the Hilbert space. The supersymmetric version of null string is given in [4, 7, 8] and for general where a null string with manifest space-time supersymmetry is given in [7] (null superstring) and one with manifest world-sheet supersymmetry is given in [4, (null spinning string).

In this paper we are going to investigate the conformal symmetry of the null spinning string at the quantum level using covariant methods and in particular the Hamiltonian BRST method [9] using the ordering presented in [3] which we find is more natural for the tensionless string discussed in [6].

The content of the paper is as follows: In section 1 we give the BRST quantization of the usual null spinning string for different numbers of supersymmetries. There, using normal ordering we reproduce for $N=0$ and $N=2$ the results obtained in [4]. Then we give the results of the BRST quantization for the physical ordering [3, 6, 9]. In section 2 we construct a null spinning string with manifest conformal invariance. Using the same methods we find negative critical dimensions for $N \neq 0$ and $d=2$ when $N=0$, as found in [9]. 


\section{The null spinning string}

The action for the null spinning string is given by 8

$$
S=\int d^{2} \sigma\left[\left(V^{a} \partial_{a} X^{\mu}+i \Psi^{i \mu} \chi^{i}\right)\left(V^{b} \partial_{b} X^{\mu}+i \Psi_{\mu}^{j} \chi^{j}\right)+i \Psi^{i \mu} V^{a} \partial_{a} \Psi_{\mu}^{i}\right]
$$

where $\mu=0, \ldots, d-1$ is a space-time index $d$ being the space-time dimension and $a=0,1$ is a world sheet index. $V^{a}$ is a weight $w=-\frac{1}{2}$ contravariant 2dimensional vector density, $\Psi^{i \mu}$ is the fermionic partner of $X^{\mu}, i=1, \ldots, N$, $N$ being the number of supersymmetries and $\chi^{i}$ is the fermionic partner of $V^{a}$. The fermions are densities of weight $w=-\frac{1}{4}$ to ensure covariance. In the $T \rightarrow 0$ limit $\chi^{i}$ arises as $\chi^{i}=V^{a} \chi_{a}^{i}$ with $\chi_{a}^{i}$ the ordinary gravitino. In (11) and in all the following relations a summation over repeated $i$ indices is assumed.

The action is invariant under the following local supersymmetry transformations

$$
\begin{aligned}
\delta X^{\mu} & =i \epsilon^{i} \Psi^{i \mu} \\
\delta \Psi^{i \mu} & =-\epsilon^{i} \partial X^{\mu}-i \epsilon^{i}\left(\Psi^{j \mu} \chi^{j}\right)-\frac{1}{2} i \Psi^{i \mu}\left(\epsilon^{j} \chi^{j}\right) \\
\delta V^{a} & =i V^{a}\left(\epsilon^{i} \chi^{i}\right) \\
\delta \chi^{i} & =\nabla \epsilon^{i}+\frac{3}{2} i\left(\epsilon^{j} \chi^{j}\right) \chi^{i}
\end{aligned}
$$

where $\epsilon=\epsilon(\sigma)$ is a density of weight $\frac{1}{4}$ and we have introduced the notation $\partial=V^{a} \partial_{a}$ and $\nabla=V^{a} \nabla_{a}$. The covariant derivative involves a connection about which it is sufficient to assume $\nabla_{a} V^{a}=0$, which thus is the "metricity condition" of our theory.

The canonical momenta derived from the action (1) are

$$
\begin{aligned}
P_{\mu} & \equiv \frac{\partial L}{\partial \dot{X}^{\mu}}=2 V^{0}\left(V^{a} \partial_{a} X_{\mu}+i \Psi_{\mu}^{i} V^{a} \chi_{a}^{i}\right) \\
\pi_{\mu}^{i} & \equiv \frac{\partial L}{\partial \dot{\Psi}^{i \mu}}=-i V^{0} \Psi_{\mu}^{i} \\
P_{\chi}^{i, a} & \equiv \frac{\partial L}{\partial \dot{\chi}_{a}^{i}}=0 \\
P_{V a} & \equiv \frac{\partial L}{\partial \dot{V}^{a}}=0 .
\end{aligned}
$$


They satisfy the usual commutation relations. The set of primary constraints is given by

$$
D_{\mu}^{i} \equiv \pi_{\mu}^{i}+i V^{0} \Psi_{\mu}^{i}=0, \quad P_{\chi}^{i, a}=0, \quad P_{V a}=0 .
$$

From the general definition of the Hamiltonian we can write

$$
H=\int d^{2} \sigma\left[\dot{X}^{\mu} P_{\mu}+\dot{\Psi}^{\mu} \pi_{\mu}-L+\lambda_{1 a}^{i} P_{\chi}^{i, a}+\lambda_{2}^{a} P_{V a}+\lambda_{3}^{i \mu} D_{\mu}^{i}\right],
$$

where the $\lambda$ 's are Lagrange multipliers. In order for the theory to be consistent all the constraints must hold for all times. This means that we have to require the following consistency conditions

$$
\dot{P}_{\chi}^{i, a}(\sigma)=\left[P_{\chi}^{i, a}(\sigma), H\right]=0 \Rightarrow S^{i,-1}(\sigma) \equiv P^{\mu} \Psi_{\mu}^{i}(\sigma)=0
$$

which means that the condition $S^{i,-1}(\sigma)=0$ is a secondary constraint. In exactly the same way we will have

$$
\begin{aligned}
\dot{P}_{V 1}(\sigma)=0 & \Rightarrow \phi^{L}(\sigma) \equiv\left(\frac{1}{V^{0}} X_{\mu}^{\prime} P^{\mu}+i \Psi^{\mu} \Psi^{\prime} \mu\right)(\sigma)=0 \\
\dot{P}_{V 0}(\sigma)=0 & \Rightarrow \frac{1}{2 V^{0}} P^{\mu} P_{\mu}-\frac{1}{V^{2}} V^{1} X^{\prime \mu} P_{\mu}-i \lambda_{3}^{i \mu} \Psi_{\mu}^{i}=0 \\
\dot{D}_{\mu}^{i}(\sigma)=0 & \Rightarrow 2 i \Psi^{i \mu} V^{1} \Psi_{\mu}^{\prime}-2 i V^{0} \lambda_{3}^{i \mu} \Psi_{\mu}^{i}=0
\end{aligned}
$$

and using these three last relations

$$
\phi^{-1}(\sigma) \equiv P^{\mu} P_{\mu}(\sigma)=0 .
$$

It is not difficult to show now that $P_{V a}(\sigma), P_{\chi}{ }^{a}(\sigma), S^{i,-1}(\sigma), \phi^{L}(\sigma)$ and $\phi^{-1}(\sigma)$ constitute a complete set of first class constraints and that the Hamiltonian is a linear combination of these. . In what follows we are going to gauge fix the field $V^{0}(\sigma)$ equal to $\frac{1}{2}$ to have a direct correspondence with previously obtained results. The remaining constraints form the following algebra

$$
\begin{aligned}
{\left[\phi^{L}(\sigma), \phi^{L}\left(\sigma^{\prime}\right)\right]_{D . B .} } & =2\left[\phi^{L}(\sigma)+\phi^{L}\left(\sigma^{\prime}\right)\right] \frac{d}{d \sigma} \delta\left(\sigma-\sigma^{\prime}\right) \\
{\left[\phi^{L}(\sigma), S^{i,-1}\left(\sigma^{\prime}\right)\right]_{D . B .} } & =2\left[S^{i,-1}(\sigma)+\frac{1}{2} S^{i,-1}\left(\sigma^{\prime}\right)\right] \frac{d}{d \sigma} \delta\left(\sigma-\sigma^{\prime}\right)
\end{aligned}
$$

\footnotetext{
${ }^{2}$ This is in fact something that we might expected since the theory is reparametrization invariant.
} 


$$
\left\{S^{i,-1}(\sigma), S^{j,-1}\left(\sigma^{\prime}\right)\right\}_{D . B .}=-\frac{i}{2}\left[\phi^{-1}(\sigma)+\phi^{-1}\left(\sigma^{\prime}\right)\right] \delta\left(\sigma-\sigma^{\prime}\right) \delta^{i j} .
$$

All other brackets are zero. Note that we replaced the original Poisson brackets with Dirac brackets to eliminate the second class constraints. This means that the canonical commutation relations will be

$$
\begin{aligned}
{\left[X^{\mu}(\sigma), P_{\nu}\left(\sigma^{\prime}\right)\right]_{D . B .} } & =\delta_{\nu}^{\mu} \delta\left(\sigma-\sigma^{\prime}\right) \\
\left\{\Psi^{i \mu}(\sigma), \Psi_{\nu}^{j}\left(\sigma^{\prime}\right)\right\}_{D . B .} & =-i \delta^{i j} \delta_{\nu}^{\mu} \delta\left(\sigma-\sigma^{\prime}\right) .
\end{aligned}
$$

To quantize the system we have to replace Dirac brackets by (anti-)commutators according to $i\{\}_{(D . B .) \pm} \rightarrow[]_{ \pm}(\hbar \equiv 1)$. Then (3) become

$$
\left[x_{m}^{\mu}, p_{n}^{\nu}\right]=i \delta_{m+n} \eta^{\mu \nu}, \quad\left[\psi_{\mu}^{i}, \psi_{\nu}^{j}\right]=\delta_{m+n}^{i j} \eta_{\mu \nu}
$$

where we have used the Fourier modes of the operators $X^{\mu}$ and $\Psi_{\mu}^{i}$. We should also note here that the mode indices of $\psi_{m}^{i \mu}$ are integral or half odd depending on the sector. In the Ramond sector they are integral and in the Neveu-Schwarz sector they are half odd. So in the quantum case the constraint algebra (2) takes the form

$$
\begin{aligned}
{\left[\phi_{m}^{-1}, \phi_{n}^{L}\right] } & =(m-n) \phi_{m+n}^{-1} \\
{\left[\phi_{m}^{L}, \phi_{n}^{L}\right] } & =(m-n) \phi_{m+n}^{L}+\left(d_{1} m^{3}+d_{2} m\right) \delta_{m+n} \\
{\left[\phi_{m}^{L}, S_{n}^{i,-1}\right] } & =\left(\frac{m}{2}-n\right) S_{m+n}^{i,-1} \\
\left\{S_{m}^{i,-1}, S_{n}^{j,-1}\right\} & =\frac{1}{2} \phi_{m+n}^{-1} \delta^{i j},
\end{aligned}
$$

where we have used the Fourier modes of the constraints which read

$$
\begin{aligned}
\phi_{m}^{-1} & =\frac{1}{2} \sum_{-\infty}^{+\infty} p_{m-k} \cdot p_{k}=0 \\
\phi_{m}^{L} & =-i \sum_{-\infty}^{+\infty}\left[k p_{m-k} \cdot x_{k}+\frac{1}{2} k \psi_{m-k}^{i} \cdot \psi_{k}^{i}\right]=0 \\
S_{m}^{i,-1} & =\frac{1}{2} \sum_{-\infty}^{+\infty} p_{m-k} \cdot \psi_{k}^{i}=0 .
\end{aligned}
$$

Note that we have included central extensions in the constraint algebra due to ordering ambiguities. The particular form of the central extensions is limited by the Jacobi identities. 
With the structure constants at hand we may write down the Hamiltonian BRST charge $\mathcal{Q}$

$$
\begin{aligned}
\mathcal{Q}= & \sum_{k}\left(\phi_{-k}^{-1} c_{k}^{-1}+\phi_{-k}^{L} c_{k}^{L}+S_{-k}^{i,-1} \gamma_{k}^{i,-1}\right) \\
- & \sum_{k, l}\left[(k-l) c_{-k}^{-1} c_{-l}^{L} b_{k+l}^{-1}+\frac{1}{2}(k-l) c_{-k}^{L} c_{-l}^{L} b_{k+l}^{L}-\left(\frac{k}{2}-l\right) c_{-k}^{L} \gamma_{-l}^{i,-1} \beta_{k+l}^{i,-1}+\right. \\
& \left.\frac{1}{4} \gamma_{-k}^{i,-1} \gamma_{-l}^{i,-1} b_{k+l}^{-1}\right]
\end{aligned}
$$

Here we have introduced the (anti-) ghosts $\left(b^{\alpha}, \beta^{i,-1}\right), c^{\alpha}, \gamma^{i,-1}$, corresponding to the constraints $\phi^{\alpha}, S^{i,-1}$, fulfilling the canonical relations $(\alpha=-1, L)$

$$
\begin{aligned}
\left\{c_{m}^{\alpha}, b_{n}^{\beta}\right\} & =\delta^{\alpha \beta} \delta_{m n} \\
{\left[\gamma_{m}^{i,-1}, \beta_{n}^{j,-1}\right] } & =\delta^{i j} \delta_{m n} .
\end{aligned}
$$

The couplings are determined by the structure constants $f^{I J K}$ of the algebra(2) according to the general prescription of [10]:

$$
\mathcal{Q}=\phi^{I} c^{J}-\frac{1}{2}(-1)^{n_{J}} f_{J K}^{I} b_{I} c^{K} c^{J}
$$

where

$$
n_{J}= \begin{cases}0 & \text { for } \phi^{J} \text { bosonic } \\ 1 & \text { for } \phi^{J} \text { fermionic }\end{cases}
$$

and the index $I$ runs over all possible constraints.

The classical nilpotency, $\mathcal{Q}^{2}=0$, is guaranteed by construction. To check the nilpotency of the quantum $\mathcal{Q}$ we use the following trick.

We begin by defining the extended constraints $\tilde{\phi}_{n}^{I}$ by the equation

$$
\tilde{\phi}_{n}^{I} \equiv\left\{b_{n}^{I}, \mathcal{Q}\right\}
$$

Using (11) we find that the extended constraints are given by the following relations

$$
\tilde{\phi}_{m}^{-1}=: \phi_{m}^{-1}:-\sum_{-\infty}^{+\infty}:(m-k) c_{-k}^{L} b_{m+k}^{-1}:
$$




$$
\begin{aligned}
\tilde{\phi}_{m}^{L}= & : \phi_{m}^{L}:+\sum_{-\infty}^{+\infty}:\left[(k-m) c_{-k}^{-1} b_{m+k}^{-1}+(k-m) c_{-k}^{L} b_{m+k}^{L}+\right. \\
& \left.+\left(\frac{m}{2}-k\right) \gamma_{-k}^{i,-1} \beta_{m+k}^{i,-1}\right]:-\alpha_{L} \delta_{m} \\
\tilde{S}_{m}^{i,-1}= & : S_{m}^{i,-1}:+\sum_{-\infty}^{+\infty}:\left[\left(\frac{k}{2}-m\right) c_{-k}^{L} \beta_{m+k}^{i,-1}-\frac{1}{2} \gamma_{-k}^{i,-1} b_{m+k}^{-1}\right]:,
\end{aligned}
$$

where : : is the ordering for which $\mathcal{Q} \mid$ phys $\rangle=0$.

It is not difficult to check that the extended constraints satisfy the same algebra as the original constraints so their algebra is the tilded version of (14)-(16).

We can now calculate the BRST anomaly using a method described in [13, 12]. There it is shown that

$$
\mathcal{Q}^{2}=\frac{1}{2} \sum_{i, j} \tilde{d}_{m}^{I J} c_{m}^{I} c_{-m}^{J}
$$

where $\tilde{d}^{I J}$ are the central extensions of the extended constraints algebra.

This means that

$$
\mathcal{Q}^{2}=\tilde{d}_{1} \sum_{m} \frac{m^{3}}{2} c_{m}^{L} c_{-m}^{L}+\tilde{d}_{2} \sum_{m} \frac{m}{2} c_{m}^{L} c_{-m}^{L} .
$$

The exact values of $\tilde{d}_{f}, f=1,2$ depend on the vacuum and ordering we have used. The simplest and safest method to determine these constants is to calculate the vacuum expectation value of the commutators (14)-(16) for the extended constraints.

We assume first that every operator with positive index annihilates the vacuum. This means that $\forall m>0$

$$
\tilde{\phi}_{m}^{-1}|0\rangle=\tilde{\phi}_{m}^{L}|0\rangle=\tilde{S}_{m}^{i,-1}|0\rangle=0 .
$$

The expectation value of the commutator (5) is

$$
\left\langle 0\left|\left[\tilde{\phi}_{m}^{L}, \tilde{\phi}_{-m}^{L}\right]\right| 0\right\rangle=2 m\left\langle 0\left|\tilde{\phi}_{0}^{L}\right| 0\right\rangle+\tilde{d}_{1} m^{3}+\tilde{d}_{2} m .
$$

In the R-sector for $m=1$ we will have

$$
2\left\langle 0\left|\tilde{\phi}_{0}^{L}\right| 0\right\rangle+\tilde{d}_{1}+\tilde{d}_{2}=\left\langle 0\left|\tilde{\phi}_{1}^{L} \tilde{\phi}_{-1}^{L}\right| 0\right\rangle=\frac{N d}{8}-4+\frac{3}{4} N .
$$


For $m=2$

$$
4\left\langle 0\left|\tilde{\phi}_{0}^{L}\right| 0\right\rangle+8 \tilde{d}_{1}+2 \tilde{d}_{2}=\left\langle 0\left|\tilde{\phi}_{2}^{L} \tilde{\phi}_{-2}^{L}\right| 0\right\rangle=d+\frac{N d}{2}-34+7 N .
$$

From the last two equations we can write

$$
\begin{array}{r}
\tilde{d}_{1}=\frac{1}{24}(4 d+N d-104+22 N) \\
\tilde{d}_{2}=\frac{1}{24}\left(-4 d+2 N d+8-4 N-48 \alpha_{L}^{R}\right),
\end{array}
$$

where $\alpha_{L}^{R}=\left\langle 0\left|\tilde{\phi}_{0}^{L}\right| 0\right\rangle$. As we can see from (18) the BRST charge can be nilpotent only if $\tilde{d}_{1}=\tilde{d}_{2}=0$ which in turn means that the following relations have to be satisfied

$$
d=\frac{104-22 N}{4+N}, \quad \alpha_{L}^{R}=-\frac{N^{2}-6 N+8}{4+N} .
$$

So in the Ramond sector we will have positive critical space-time dimensions for the following numbers of supersymmetries

\begin{tabular}{|c||c|c|}
\hline \multicolumn{3}{|c|}{ Ramond Sector } \\
\hline $\mathrm{N}$ & Critical dimension & $\alpha_{L}^{R}$ \\
\hline \hline 0 & 26 & -2 \\
\hline 2 & 10 & 0 \\
\hline 4 & 2 & 0 \\
\hline
\end{tabular}

In exactly the same manner we can find that in the NS-sector we will have the following relations

$$
d=\frac{104-22 N}{4+N}, \quad \alpha_{L}^{N S}=\frac{N^{2}-16}{2(4+N)} .
$$

So in the Neveu-Schwarz sector we will have the following table

\begin{tabular}{|c||c|c|}
\hline \multicolumn{3}{|c|}{ Neveu-Schwarz Sector } \\
\hline $\mathrm{N}$ & Critical dimension & $\alpha_{L}^{N S}$ \\
\hline \hline 0 & 26 & -2 \\
\hline 2 & 10 & -1 \\
\hline 4 & 2 & 0 \\
\hline
\end{tabular}


Note that the results for $N=0,2$ are those obtained in [⿴囗十). We should also remark that the $N=4$ case gives the same critical dimension, $d=2$, with the $N=2$ formulation of the usual $T \neq 0$ spinning string.

According to arguments presented in [6] however the vacuum suitable for tensionless is not the one annihilated by the positive modes of the operators but the one annihilated by the momenta

$$
p_{m}^{\mu}|0\rangle=0 \quad \forall m .
$$

Following the prescription of [11, 9], we will take the ket states to be built from our vacuum of choice, $|0\rangle_{p}$, and the bra states to be built from ${ }_{x}\langle 0|$ satisfying ${ }_{x}\langle 0 \mid 0\rangle_{p}=1$. For this vacuum and from the requirement that the BRST charge (17) should annihilate the vacuum, we can find requirements for the ghost part of the vacuum. Doing this we find that the vacuum has to satisfy the following conditions $\forall m, i$

$$
\begin{gathered}
p_{m}^{\mu}|0\rangle=b_{m}^{-1}|0\rangle=\beta_{m}^{i,-1}|0\rangle=0 \\
\langle 0| x_{m}^{\mu}=\langle 0| c_{m}^{-1}=\langle 0| \gamma_{m}^{i,-1}=0 .
\end{gathered}
$$

As can be seen from the previous relations the condition $p_{m}^{\mu}|0\rangle=0$ does not specify how the $\psi_{m}^{i \mu}$ operators will act on this vacuum. We do not like to have a condition like $\psi_{m}^{i \mu}|0\rangle=0, \forall m$ on the vacuum since this would destroy all the fermionic creation operators. Choosing $\psi_{m}^{i \mu}|0\rangle=0, \forall m>0$ will give us the following conditions

$$
\begin{array}{r}
\psi_{m}^{i \mu}|0\rangle=c_{m}^{L}|0\rangle=b_{m}^{L}|0\rangle=0 \\
\langle 0| \psi_{-m}^{i \mu}=\langle 0| c_{-m}^{L}=\langle 0| b_{-m}^{L}=0 .
\end{array}
$$

With this choice of vacuum it is not difficult to find that the commutator (9) will give for $N \neq 0$ 目

$$
\begin{aligned}
\tilde{d}_{1} & =\frac{1}{24}(N d-52) \\
\tilde{d}_{2}^{R} & =\frac{1}{24}\left(2 N d+4-48 \alpha_{L}^{R}\right), \quad \text { for the R-sector, } \\
\tilde{d}_{2}^{L S} & =\frac{1}{24}\left(-N d+4-48 \alpha_{L}^{N S}\right), \quad \text { for the NS-sector. }
\end{aligned}
$$

\footnotetext{
${ }^{3}$ For $N=0$ it can be shown that $\tilde{d}_{1}=\tilde{d}_{2}=0$ and thus that $\mathcal{Q}^{2}=0$, independent of the dimension $d$.
} 
So in this case we will have the following

\begin{tabular}{|c||c|c|c|}
\hline \multicolumn{4}{|c|}{ Physical Ordering } \\
\hline $\mathrm{N}$ & Critical dimension & $\alpha_{L}^{R}$ & $\alpha_{L}^{N S}$ \\
\hline \hline 1 & 52 & $\frac{9}{4}$ & -1 \\
\hline 2 & 26 & $\frac{9}{4}$ & -1 \\
\hline 4 & 13 & $\frac{9}{4}$ & -1 \\
\hline 13 & 4 & $\frac{9}{4}$ & -1 \\
\hline 26 & 2 & $\frac{9}{4}$ & -1 \\
\hline 52 & 1 & $\frac{9}{4}$ & -1 \\
\hline
\end{tabular}

\section{The conformal spinning string}

Let us return to the action of the null spinning string (1). It is not difficult to check that the action is invariant under the group of space-time conformal transformations

(i) Lorentz transformations

$$
\begin{aligned}
\delta_{\omega} X^{\mu} & =\omega_{\nu}^{\mu} X^{\nu} \\
\delta_{\omega} \Psi^{i \mu} & =\omega_{\nu}^{\mu} \Psi^{i \nu} \\
\delta_{\omega} V^{a} & =0 \\
\delta_{\omega} \chi_{a}^{i} & =0
\end{aligned}
$$

(ii) Translations

$$
\begin{aligned}
\delta_{l} X^{M} & =l^{\mu} \\
\delta_{l} \Psi^{i \mu} & =0 \\
\delta_{l} V^{a} & =0 \\
\delta_{l} \chi_{a}^{i} & =0
\end{aligned}
$$

(iii) Special conformal transformations

$$
\begin{aligned}
\delta_{b} X^{\mu} & =(b \cdot X) X^{\mu}-\frac{1}{2} X^{2} b^{\mu} \\
\delta_{b} \Psi^{i \mu} & =\frac{1}{2}(b \cdot X) \Psi^{i \mu} \\
\delta_{b} V^{a} & =-(b \cdot X) V^{a} \\
\delta_{b} \chi_{a}^{i} & =\frac{1}{2}(b \cdot X) \chi_{a}^{i}
\end{aligned}
$$


(iv) Dilatations

$$
\begin{aligned}
\delta_{a} X^{\mu} & =a X^{\mu} \\
\delta_{a} \Psi^{i \mu} & =\frac{a}{2} \Psi^{i \mu} \\
\delta_{a} V^{a} & =a V^{a} \\
\delta_{a} \chi_{a}^{i} & =\frac{a}{2} \chi_{a}^{i}
\end{aligned}
$$

The isomorphism $C_{d-1,1} \simeq O(d, 2)$ for $d \geq 3$ makes it possible to construct a theory in two extra dimensions such that the previous model corresponds to a particular gauge fixing of the latter and the conformal symmetry is manifest and linearly realized [2, 12, 9]. This conformal spinning string action can be given by

$$
\begin{aligned}
S= & \int d^{2} \sigma\left\{\left[V^{a}\left(\partial_{a}+W_{a}\right) X^{A}+i \Psi^{i A} \chi^{i}\right]\left[V^{b}\left(\partial_{b}+W_{b}\right) X_{A}+i \Psi_{A}^{j} \chi^{j}\right]+\right. \\
& \left.i \Psi^{i A} V^{a} \partial_{a} \Psi_{A}^{i}+i A^{i j} \Psi^{i A} \Psi_{A}^{j}+\Phi X^{A} X_{A}\right\}
\end{aligned}
$$

where $A=0, \ldots, d+1$ and the new metric has the form

$$
\eta_{A B}=\left(\begin{array}{lll}
\eta_{\mu \nu} & 0 & 0 \\
0 \ldots 0 & 1 & 0 \\
0 \ldots 0 & 0- & 1
\end{array}\right) .
$$

$W_{a}$ is the gauge field for scale transformations, $A^{i j}$ and $\Phi$ are Lagrange multiplier fields.

We can check that by imposing two gauge fixing conditions $P^{+}=0$, $X^{+}=1$ the generators of the Lorentz transformations in the extended space become the generators of the conformal group in the original space. Thus rotations in the extended space correspond to conformal transformations in the original space.

Going to the Hamiltonian formulation we find in exactly the same manner that the Hamiltonian is again a linear combination of the constraints. In addition to the original constraints (8)-(10) we will have four new ones which in Fourier modes can be written as follows

$$
\phi_{m}^{0}=\frac{1}{2} \sum_{-\infty}^{+\infty} p_{m-k} \cdot x_{k}=0
$$




$$
\begin{aligned}
\phi_{m}^{1} & =\frac{1}{2} \sum_{-\infty}^{+\infty} x_{m-k} \cdot x_{k}=0 \\
S_{m}^{i, 1} & =\frac{1}{2} \sum_{-\infty}^{+\infty} x_{m-k} \cdot \psi_{k}^{i}=0 \\
\phi_{m}^{i j} & =\frac{1}{2} \sum_{-\infty}^{+\infty} \psi_{m-k}^{i} \cdot \psi_{k}^{j}=0
\end{aligned}
$$

where $i, j=1, \ldots, N$ and $i<j$ in the last constraint. Note that if one discards $\phi_{m}^{L}$, the remaining constraints will describe the conformal spinning particle presented in [14].

The constraint algebra with the central extensions included will be given by

$$
\begin{aligned}
{\left[\phi_{m}^{L}, \phi_{n}^{i j}\right] } & =-n \phi_{m+n}^{i j} \\
{\left[S_{m}^{i,-1}, \phi_{n}^{\alpha \beta}\right] } & =\frac{1}{2} S_{m+n}^{\beta,-1} \delta^{i \alpha}-\frac{1}{2} S_{m+n}^{\alpha,-1} \delta^{i \beta} \\
{\left[\phi_{m}^{i j}, \phi_{n}^{\alpha \beta}\right] } & =\frac{1}{2} \phi_{m+n}^{i \beta} \delta^{\alpha j}-\frac{1}{2} \phi_{m+n}^{\alpha j} \delta^{i \beta}+\frac{1}{2} \phi_{m+n}^{\beta j} \delta^{i \alpha}-\frac{1}{2} \phi_{m+n}^{i \alpha} \delta^{j \beta} \\
{\left[\phi_{m}^{1}, \phi_{n}^{-1}\right] } & =2 i \phi_{m+n}^{0}+2\left(i d_{4}+i d_{3} m\right) \delta_{m+n} \\
{\left[\phi_{m}^{1}, \phi_{n}^{L}\right] } & =(m+n) \phi_{m+n}^{1} \\
{\left[\phi_{m}^{1}, S_{n}^{i,-1}\right] } & =i S_{m+n}^{i, 1} \\
{\left[\phi_{m}^{-1}, \phi_{n}^{0}\right] } & =-i \phi_{m+n}^{-1} \\
{\left[\phi_{m}^{0}, \phi_{n}^{L}\right] } & =m \phi_{m+n}^{0}+\left(d_{3} m^{2}+d_{4} m\right) \delta_{m+n} \\
{\left[\phi_{m}^{0}, S_{n}^{i,-1}\right] } & =\frac{i}{2} S_{m+n}^{i,-1} \\
{\left[\phi_{m}^{1}, \phi_{n}^{0}\right] } & =i \phi_{m+n}^{1} \\
{\left[\phi_{m}^{-1}, S_{n}^{i, 1}\right] } & =-i S_{m+n}^{i,-1} \\
{\left[\phi_{m}^{L}, S_{n}^{i, 1}\right] } & =-\left(\frac{m}{2}+n\right) S_{m+n}^{i, 1} \\
\left\{S_{m}^{i,-1}, S_{n}^{j, 1}\right\} & =\frac{1}{2} \phi_{m+n}^{0} \delta^{i j}-\frac{i}{2} \phi_{m+n}^{i j}+\left(\frac{d_{4}}{2}-m d_{3}\right) \delta^{i j} \delta_{m+n} \\
{\left[S_{m}^{i,-1}, \phi_{n}^{\alpha \beta}\right] } & =\frac{1}{2} S_{m+n}^{\beta, 1} \delta^{i \alpha}-\frac{1}{2} S_{m+n}^{\alpha, 1} \delta^{i \beta}
\end{aligned}
$$




$$
\begin{aligned}
{\left[\phi_{m}^{0}, S_{n}^{i, 1}\right] } & =-\frac{i}{2} S_{m+n}^{i, 1} \\
\left\{S_{m}^{i, 1}, S_{n}^{j, 1}\right\} & =\frac{1}{2} \phi_{m+n}^{1} \delta^{i j} \\
{\left[\phi_{m}^{0}, \phi_{n}^{0}\right] } & =-i m d_{3} \delta_{m+n} .
\end{aligned}
$$

All the other commutators except (4)-(77) vanish.

The structure constants obtained here can be inserted into the relation (12) to find the BRST charge which is rather complicated this time due to the large number of constraints

$$
\begin{aligned}
\mathcal{Q}= & \sum_{k}\left(\phi_{-k}^{1} c_{k}^{1}+\phi_{-k}^{0} c_{k}^{0}+\phi_{-k}^{-1} c_{k}^{-1}+\phi_{-k}^{L} c_{k}^{L}\right) \\
+ & \sum_{k}\left(S_{-k}^{i,-1} \gamma_{k}^{i,-1}+S_{-k}^{i, 1} \gamma_{k}^{i, 1}+\sum_{i<j} \phi_{-k}^{i j} c_{k}^{i j}\right) \\
+ & \sum_{k, l}\left[-2 i c_{-k}^{1} c_{-l}^{-1} b_{k+l}^{0}-i c_{-k}^{1} c_{-l}^{0} b_{k+l}^{1}+i c_{-k}^{-1} c_{-l}^{0} b_{k+l}^{-1}-\right. \\
& (k+l) c_{-k}^{1} c_{-l}^{L} b_{k+l}^{1}-(k-l) c_{-k}^{-1} c_{-l}^{L} b_{k+l}^{-1}-k c_{-k}^{0} c_{-l}^{L} b_{k+l}^{0}- \\
& \frac{1}{2}(k-l) c_{-k}^{L} c_{-l}^{L} b_{k+l}^{L}+\left(\frac{k}{2}-l\right) c_{-k}^{L} \gamma_{-l}^{i,-1} \beta_{k+l}^{i,-1}-\frac{1}{4} \gamma_{-k}^{i,-1} \gamma_{-l}^{i,-1} b_{k+l}^{-1}+ \\
& i c_{-k}^{1} \gamma_{-l}^{i,-1} \beta_{k+l}^{i, 1}+\frac{i}{2} c_{-k}^{0} \gamma_{-l}^{i,-1} \beta_{k+l}^{i,-1}-i c_{-k}^{-1} \gamma_{-l}^{i, 1} \beta_{k+l}^{i,-1}- \\
& \left(\frac{k}{2}+l\right) c_{-k}^{L} \gamma_{-l}^{i, 1} \beta_{k+l}^{i, 1}-\frac{i}{2} c_{-k}^{0} \gamma_{-l}^{i, 1} \beta_{k+l}^{i, 1}-\frac{1}{2} \gamma_{-k}^{i,-1} \gamma_{-l}^{i, 1} b_{k+l}^{0}- \\
& \frac{1}{4} \gamma_{-k}^{i, 1} \gamma_{-l}^{i, 1} b_{k+l}^{1}+\frac{i}{2} \gamma_{-k}^{i,-1} \gamma_{-l}^{j, 1} b_{k+l}^{i j}-\frac{i}{2} \gamma_{-k}^{i,-1} \gamma_{-l}^{j, 1} b_{k+l}^{j i}+ \\
& l c_{-k}^{L} c_{-l}^{i j} b_{k+l}^{i j}-\frac{1}{2} c_{-k}^{i j} c_{-l}^{j \alpha} b_{k+l}^{i \alpha}+\frac{1}{2} c_{-k}^{i j} c_{-l}^{\alpha i} b_{k+l}^{\alpha j}+ \\
& \frac{1}{2} c_{-k}^{i j} c_{-l}^{i \alpha} b_{k+l}^{\alpha j}-\frac{1}{2} c_{-k}^{i j} c_{-l}^{\alpha j} b_{k+l}^{i \alpha}-\frac{1}{2} \gamma_{-k}^{i,-1} c_{-l}^{i \alpha} \beta_{k+l}^{\alpha,-1}+ \\
& \left.\frac{1}{2} \gamma_{-k}^{i,-1} c_{-l}^{\alpha i} \beta_{k+l}^{\alpha,-1}-\frac{1}{2} \gamma_{-k}^{i, 1} c_{-l}^{i \alpha} \beta_{k+l}^{\alpha, 1}+\frac{1}{2} \gamma_{-k}^{i, 1} c_{-l}^{\alpha i} \beta_{k+l}^{\alpha, 1}\right] .
\end{aligned}
$$

We can check the nilpotency of $\mathcal{Q}$ with the use of the extended constraints. Due to (13) and (27) we will have

$$
\begin{aligned}
\tilde{\phi}_{m}^{-1}= & : \phi_{m}^{-1}:+\sum_{-\infty}^{+\infty}:\left[2 i c_{-k}^{1} b_{m+k}^{0}+i c_{-k}^{0} b_{m+k}^{-1}-(m-k) c_{-k}^{L} b_{m+k}^{-1}-\right. \\
& \left.i \gamma_{-k}^{i, 1} \beta_{m+k}^{i,-1}\right]:
\end{aligned}
$$




$$
\begin{aligned}
& \tilde{\phi}_{m}^{L}=: \phi_{m}^{L}:+\sum_{-\infty}^{+\infty}:\left[(k+m) c_{-k}^{1} b_{k+m}^{1}+(k-m) c_{-k}^{-1} b_{m+k}^{-1}+\right. \\
& k c_{-k}^{0} b_{m+k}^{0}+(k-m) c_{-k}^{L} b_{m+k}^{L}+k c_{-k}^{i j} b_{m+k}^{i j}+ \\
& \left.\left(\frac{m}{2}-k\right) \gamma_{-k}^{i,-1} \beta_{m+k}^{i,-1}-\left(\frac{m}{2}+k\right) \gamma_{-k}^{i, 1} \beta_{m+k}^{i, 1}\right]:-\alpha_{L} \delta_{m} \\
& \tilde{S}_{m}^{i,-1}=: S_{m}^{i,-1}:+\sum_{-\infty}^{+\infty}:\left[\left(\frac{k}{2}-m\right) c_{-k}^{L} \beta_{m+k}^{i,-1}-\frac{1}{2} \gamma_{-k}^{i,-1} b_{m+k}^{-1}-\frac{1}{2} c_{-k}^{i \alpha} \beta_{m+k}^{\alpha,-1}+\right. \\
& \frac{1}{2} c_{-k}^{\alpha i} \beta_{m+k}^{\alpha,-1}+i c_{-k}^{1} \beta_{m+k}^{i, 1}+\frac{i}{2} c_{-k}^{0} \beta_{m+k}^{i,-1}- \\
& \left.\frac{1}{2} \gamma_{-k}^{i, 1} b_{m+k}^{0}+\frac{i}{2} \gamma_{-k}^{j, 1} b_{m+k}^{i j}-\frac{i}{2} \gamma_{-k}^{j, 1} b_{m+k}^{j i}\right] \text { : } \\
& \tilde{\phi}_{m}^{0}=: \phi_{m}^{0}:+\sum_{-\infty}^{+\infty}:\left[i c_{-k}^{1} b_{m+k}^{1}-i c_{-k}^{-1} b_{m+k}^{-1}-m c_{-k}^{L} b_{m+k}^{0}-\right. \\
& \left.\frac{i}{2} \gamma_{-k}^{i, 1} \beta_{m+k}^{i, 1}+\frac{i}{2} \gamma_{-k}^{i,-1} \beta_{m+k}^{i,-1}\right]:-\alpha_{0} \delta_{m} \\
& \tilde{\phi}_{m}^{1}=: \phi_{m}^{1}:+\sum_{-\infty}^{+\infty}:\left[-2 i c_{-k}^{-1} b_{m+k}^{0}-i c_{-k}^{0} b_{m+k}^{1}-(m+k) c_{-k}^{L} b_{m+k}^{1}+\right. \\
& \left.i \gamma_{-k}^{i,-1} \beta_{m+k}^{i, 1}\right]: \\
& \tilde{S}_{m}^{i, 1}=: S_{m}^{i, 1}:+\sum_{-\infty}^{+\infty}:\left[-\left(\frac{k}{2}+m\right) c_{-k}^{L} \beta_{m+k}^{i, 1}-\frac{1}{2} \gamma_{-k}^{i, 1} b_{m+k}^{1}-\frac{1}{2} c_{-k}^{i \alpha} \beta_{m+k}^{\alpha, 1}+\right. \\
& \frac{1}{2} c_{-k}^{\alpha i} \beta_{m+k}^{\alpha, 1}-i c_{-k}^{-1} \beta_{m+k}^{i,-1}-\frac{i}{2} c_{-k}^{0} \beta_{m+k}^{i, 1}- \\
& \left.\frac{1}{2} \gamma_{-k}^{i,-1} b_{m+k}^{0}-\frac{i}{2} \gamma_{-k}^{j,-1} b_{m+k}^{i j}+\frac{i}{2} \gamma_{-k}^{j,-1} b_{m+k}^{j i}\right]: \\
& \tilde{\phi}_{m}^{i j}=: \phi_{m}^{i j}:+\sum_{-\infty}^{+\infty}:\left[-m c_{-k}^{L} b_{m+k}^{i j}+\frac{1}{2} c_{-k}^{i \alpha} b_{m+k}^{\alpha j}+\frac{1}{2} c_{-k}^{i \alpha} b_{m+k}^{j \alpha}-\right. \\
& \frac{1}{2} c_{-k}^{\alpha j} b_{m+k}^{i \alpha}-\frac{1}{2} c_{-k}^{\alpha j} b_{m+k}^{\alpha i}-\frac{1}{2} \gamma_{-k}^{i,-1} \beta_{m+k}^{j,-1}+ \\
& \left.\frac{1}{2} \gamma_{-k}^{j,-1} \beta_{m+k}^{i,-1}-\frac{1}{2} \gamma_{-k}^{i, 1} \beta_{m+k}^{j, 1}+\frac{1}{2} \gamma_{-k}^{j, 1} \beta_{m+k}^{i, 1}\right]: \text {. }
\end{aligned}
$$

They satisfy the same algebra as the original constraints. This means that (17)

$$
\mathcal{Q}^{2}=\tilde{d}_{1} \sum_{m} \frac{m^{3}}{2} c_{m}^{L} c_{-m}^{L}
$$




$$
\begin{aligned}
& +\tilde{d}_{2} \sum_{m} \frac{m}{2} c_{m}^{L} c_{-m}^{L} \\
& +\tilde{d}_{3} \sum_{m}\left(-i \frac{m}{2} c_{m}^{0} c_{-m}^{0}+m^{2} c_{m}^{0} c_{-m}^{L}+2 i m c_{m}^{1} c_{-m}^{-1}-m \gamma_{m}^{i,-1} \gamma_{-m}^{i, 1}\right) \\
& +\tilde{d}_{4} \sum_{m}\left(m c_{m}^{0} c_{-m}^{L}+2 i c_{m}^{1} c_{-m}^{-1}+\frac{1}{2} \gamma_{m}^{i,-1} \gamma_{-m}^{i, 1}\right) .
\end{aligned}
$$

The only thing that remains now is to calculate the values of the constants $\tilde{d}_{f}=1, \ldots, 4$ for the vacuum and ordering introduced introduced in the previous section. In this case the condition $p_{m}^{A}|0\rangle=0 \quad \forall A$ together with the requirement that the $\mathrm{BRST}$ charge (27) gives the following consistency conditions $\forall m$

$$
\begin{gathered}
p_{m}^{A}|0\rangle=c_{m}^{1}|0\rangle=\gamma_{m}^{i, 1}|0\rangle=b_{m}^{-1}|0\rangle=\beta_{m}^{i,-1}|0\rangle=0 \\
\langle 0| x_{m}^{A}=\langle 0| c_{m}^{-1}=\langle 0| \gamma_{m}^{i,-1}=\langle 0| b_{m}^{1}=\langle 0| \beta_{m}^{i, 1}=0 .
\end{gathered}
$$

We find the values of $\tilde{d}_{3}$ and $\tilde{d}_{4}$ by calculating the expectation value of the commutator $\left\langle 0\left|\left[\tilde{\phi}_{m}^{0}, \tilde{\phi}_{-m}^{L}\right]\right| 0\right\rangle$. Indeed we have

$$
\begin{gathered}
\left\langle 0\left|\left[\tilde{\phi}_{m}^{0}, \tilde{\phi}_{-m}^{L}\right]\right| 0\right\rangle=m\left\langle 0\left|\tilde{\phi}_{0}^{0}\right| 0\right\rangle+\tilde{d}_{3} m^{2}+\tilde{d}_{4} m \\
\Rightarrow 0=m \alpha_{0}+\tilde{d}_{3} m^{2}+\tilde{d}_{4} m=0 \\
\Rightarrow \tilde{d}_{3}=0, \quad \tilde{d}_{4}=-\alpha_{0} .
\end{gathered}
$$

But from (31) for a Hermitian BRST charge $\mathcal{Q}$ we will have

$$
\alpha_{0} \equiv\left\langle 0\left|\tilde{\phi}_{0}^{0}\right| 0\right\rangle=-\frac{i}{4}[d-2+2 N] \lim _{K \rightarrow+\infty}\left(\sum_{k=-K}^{+K} 1\right) .
$$

The BRST charge is nilpotent when all the constants $\tilde{d}_{f}=1, \ldots, 4$ are equal to 0 . In particular we must have

$$
\tilde{d}_{4}=0 \Rightarrow d=2-2 N \text {. }
$$

This last relation shows us that we cannot have a positive critical dimension for $N \neq 0$ irrespective of how $\psi_{A}^{i}$ acts on vacuum. Note also that (37) gives $d=2$ for $N=0$ as obtained in 9 .

In this article we carried the techniques developed in [9] for the conformal string, to the $T \rightarrow 0$ limit of the spinning string. Faced with the anomaly 
(36), it would be interesting to see if we could use the philosophy of [6] which led to a "topological state space" result. The natural generalization in our case would be to require that $\mathcal{Q}^{2}=0$ only on physical states. This is a topic of future investigations. Another interesting problem is to see if it is possible to apply the same techniques to the space-time superstring case and to investigate if this theory is equivalent to the one presented here.

Acknowledgements:I would like to thank Ulf Lindström and Rikard von Unge for useful comments and fruitful discussions. 


\section{References}

[1] A. Schild, Phys.Rev.D 16 (1977) 1722.

[2] A. Karlhede and U. Lindström, Class.Quant.Grav. 3 (1986) L73.

[3] F. Lizzi, B. Rai, G. Sparano and A. Srivastava, Phys.Lett. 182B (1986) 326.

[4] J. Gamboa, C. Ramirez and M. Ruiz-Altaba, Phys.Lett. 225B (1989) 335.

[5] J. Isberg, U. Lindström and B. Sundborg, Phys. Lett., 293B (1992) 321.

[6] J. Isberg, U. Lindström B. Sundborg and G. Theodoridis, Nucl. Phys. B411 (1994) 122.

[7] U. Lindström, B. Sundborg and G. Theodoridis, Phys.Lett. 253B (1991) 319.

[8] U. Lindström, B. Sundborg and G. Theodoridis, Phys.Lett. 258B (1991) 331.

[9] H. Gustafsson, U. Lindström, P. Saltsidis, B. Sundborg and R.v. Unge, "Hamiltonian BRST Quantization of the Conformal String", Stockholm University preprint USITP-94-08 (1994), to appear in Nucl. Phys. B.

[10] E.S. Fradkin and G.A. Vilkovisky, Phys. Lett. 55B (1975) 224; I.A. Batalin and G.A. Vilkovisky, Phys. Lett. 69B (1977) 343.

[11] S. Hwang and R. Marnelius, Nucl. Phys. B315 (1989) 638.

[12] J. Isberg, "Tensionless Strings with Manifest Space-Time Conformal Invariance", Stockholm University preprint USITP-92-10 (1992).

[13] R. Marnelius, Nucl. Phys. B294 (1987) 685.

[14] U. Mårtensson, Int. J. Mod. Phys. A8 (1993) 5305. 